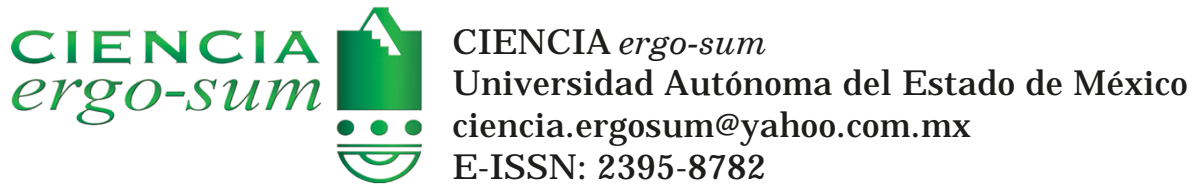

\title{
F actores determinantes de la reputación gubernamental: una ponderación a través del algoritmo de Saaty
}

Moreno Manzo, J oanna Margarita; Navarro Chávez, J osé César Lenin

Factores determinantes de la reputación gubernamental: una ponderación a través del algoritmo de Saaty

CIE NCIA ergo-sum, vol. 27, núm. 1, marzo-junio 2020|e71

Universidad Autónoma del Estado de México, México

Esta obra está bajo una Licencia Creative Commons Atribución-NoComercial-SinDerivar 4.0 Internacional .

Moreno Manzo, J . M. y Navarro Chávez, J . C. L . (2020). Factores determinantes de la reputación gubernamental: una ponderación a través del algoritmo de Saaty. CIENCIA ergo-sum, 27(1). https://doi.org/10.30878/ces.v27n1a3 


\title{
Factores determinantes de la reputación gubernamental: una ponderación a través del algoritmo de Saaty
}

Government reputation elements: A ranking through the Saaty's algorithm

Joanna Margarita Moreno Manzo

Universidad Michoacana de San Nicolás de Hidalgo, México

joannammm@gmail.com

Recepción: 08 de febrero de 2018

Aprobación: 29 de noviembre de 2018

José César Lenin Navarro Chávez

Universidad Michoacana de San Nicolás de Hidalgo, México

cesar.navarro@umich.mx

\section{RESUMEN}

Los bajos niveles de aprobación tanto de gobiernos como de gobernantes en el ámbito internacional implican un problema de legitimidad política. Parte del éxito de una organización depende de su reputación y de cómo es vista por sus stakeholders. En este contexto, se plantea encontrar los factores que determinan la reputación gubernamental para lo cual se instrumentó un método de ponderación de variables a través del algoritmo de Saaty con base en la revisión de la literatura de más de treinta obras. De los resultados obtenidos, se encontró que el desempeño gubernamental, el comportamiento ético, el liderazgo de las autoridades y la comunicación gubernamental son las variables que más incidieron en la reputación gubernamental.

Palabras ClaVe: reputación, gobierno, Saaty, legitimidad, municipio.

\begin{abstract}
The low levels of approval from both governments and authorities around the world reflects a problem of political legitimacy. The success of an organization relies partially on its reputation, on how the organization is perceived by its stakeholders. The objective of the study is to discover the main elements of government reputation. In order to accomplished it the analytical hierarchy process (Saat's Algorithm) was implemented based on the literature review, including more than 30 papers. We found that government performance, ethical behavior, the leadership of the authorities and government communication are the most influential variables of government reputation.
\end{abstract}

KEYWORDS: reputation, government, Saaty, legitimacy, municipality.

\section{INTRODUCCIÓN}

Los bajos niveles de aprobación de gobiernos y gobernantes no es un problema exclusivo de México. De acuerdo con la Encuesta Mundial de Valores, en promedio en el mundo $50.7 \%$ de las personas confía poco o nada en su gobierno nacional y de manera similar 57\% confía poco o nada en el congreso o parlamento (WVS, 2015). Esta misma encuesta, en su versión para Latinoamérica, muestra que para 2012 en México $61 \%$ de las personas confía poco o nada en su gobierno; $73 \%$ de los mexicanos confía poco o nada en el Congreso y $77.6 \%$ confía poco o nada en la burocracia. Estos datos nos ponen de relieve que los niveles de desconfianza que hay ante estas instituciones públicas en México está por encima del promedio mundial (WVS, 2012).

En la misma línea que los resultados de la Encuesta Mundial de Valores, en el sexenio 2012-2018 en México se han observado los niveles más bajos de aprobación del gobierno federal: pasó de una aprobación de $66 \%$ en agosto de 2007 a 29\% en agosto de 2016. En el caso de los gobernadores, las cifras tienen un comportamiento similar: el promedio de aprobación del trabajo de los gobernadores pasó de un $71 \%$ en agosto de 2007 a un 36\% en 2016; en los presidentes municipales, el promedio de aprobación en el país pasó de 56\% en 2007 a 38\% en 2016 (Consulta Mitofsky, 2016). 
Se puede afirmar que el éxito de una organización depende de su reputación, particularmente en los niveles de confianza, satisfacción y en cómo la organización es vista por sus stakeholders. La reputación se define con base en las percepciones (Eisseneger, 2008) o experiencias (Canel, 2014) de los stakeholders, y por lo tanto es un intangible multifactorial; sin embargo, existe poca claridad sobre los factores que determinan la reputación de un gobierno municipal. Al respecto, este trabajo tiene como objetivo encontrar una definición de reputación gubernamental, explicar los principales beneficios de gestionar una reputación positiva, explicar cuáles son esos factores que la determinan y, por último, a través de un proceso analítico jerárquico, determinar la ponderación de cada una de los factores determinantes de la reputación gubernamental.

\section{ReVisión De LA LITERATURa}

\section{1. La reputación gubernamental como un intangible del sector público}

Cahill et al. (2004) afirman que la reputación es el reconocimiento de otras personas respecto a algunas características o habilidades; para Fombrun (1996) es una estimación general de una organización por parte de sus stakeholders, la cual es expresada por las relaciones afectivas de los clientes, inversionistas, empleados y del público en general. Por lo tanto, los stakeholders o actores interesados, es decir, aquellos grupos o individuos que sin su apoyo la organización dejaría de existir y cuyo objetivo es influir o que también son influenciados por la organización, son vitales para construir la reputación de la organización (Luoma-aho, 2008).

Fombrun (1996) establece que la reputación no sólo es multifactorial, sino que además depende de múltiples sujetos y de la imagen que tienen éstos de la corporación; en relación con las organizaciones del sector público, los stakeholders tienen varios roles; por ejemplo, un empleado no deja de ser un usuario de los servicios públicos y a la vez un ciudadano que vota en las elecciones por la continuidad o no del gobierno.

Canel (2014) argumenta que el sector público trata con más servicios intangibles a diferencia de los productos o servicios que ofrecen las organizaciones privadas tales como proveer seguridad, incrementar los niveles de educación o impartir justicia. Para esta autora el carácter intangible de muchos de los servicios públicos hace la experiencia de los ciudadanos más compleja, ya que no todos reciben el servicio en el mismo momento ni en calidad ni en cantidad.

A diferencia de las organizaciones del sector privado, las organizaciones públicas tienen la obligación legal de la transparencia y la rendición de cuentas, sin dejar de lado que existe dificultad para que los gobiernos planeen a largo plazo ante la posible alternancia (Canel, 2014).

Luoma-aho (2007) enuncia algunas de las características que tienen las organizaciones del sector público y que son determinantes para la reputación de dicha organización: el ambiente político, la existencia de un marco jurídico que limita el actuar de las autoridades, la obligación de rendir cuentas y los múltiples stakeholders.

La reputación se puede definir también como "un record de acciones pasadas, y su reflejo en la habilidad de la organización para satisfacer las necesidades y las expectativas de sus stakeholders” (Luoma-aho y Makikangas, 2014: 41).

La reputación en las organizaciones del sector público se compone de tres dimensiones o mundos: el primer mundo es el de la verdad, de lo objetivo, es decir, la dimensión funcional de la reputación. El segundo mundo es el de lo normativo o lo bueno y es llamado la dimensión social de la reputación. Por último, el mundo de los subjetivo o de lo bonito, el cual se convierte en la dimensión expresiva de la reputación (Eisenegger e Imhof, 2008).

Partiendo de las definiciones sobre reputación y reputación en el sector público de algunos autores (Fombrun, 1996; Luoma-aho, 2007; Canel, 2014; Luoma-aho y Makikangas, 2014; Canel, 2017), se propone el siguiente concepto: la reputación gubernamental es la valoración colectiva de múltiples actores de interés o

stakeholders hacia un gobierno que se basa en experiencias personales, en percepciones o experiencias mediadas, por lo que es comunicable, y también se basa en las expectativas que tienen los stakeholders sobre el gobierno; es continuada, ya que depende de experiencias o percepciones pasadas, presentes o futuras del gobierno, 
implica una comparación con otros gobiernos y genera actitudes y comportamientos por parte de los stakeholders hacia la organización.

\subsection{Beneficios de la reputación gubernamental}

Vale la pena determinar los factores determinantes de la reputación y gestionarla por los beneficios que acarrea para el sector público y para la sociedad, los cuales podrían agruparse en económicos, políticos y sociales.

La reputación se puede concebir como un círculo virtuoso que atrae inversión y nuevos negocios al lugar generando empleos; incluso, es posible que gracias a la percepción positiva del gobierno que tienen los ciudadanos se incremente la recaudación de impuestos al considerar que serán bien utilizados (Cahill et al., 2004; Arana y Caicedo, 2013).

Hoy en día las ciudades compiten con otras en la atracción de turismo, empresas, residentes, inversionistas, emprendedores e incluso estudiantes universitarios. Una buena reputación del gobierno y de la ciudad sin duda es un factor determinante para la elección (Kizil y Atalan, 2015).

Para Bannister et al. (2003), los gobiernos con una buena reputación mantienen e incrementan potencialmente la inversión privada en su región; además de ofrecer buena imagen para los líderes políticos y los partidos políticos, se puede reducir a su vez la imagen negativa de las instituciones políticas y se disminuye la apatía de los ciudadanos hacia la institución o gobierno fortaleciendo la participación ciudadana en asuntos políticos.

Una reputación positiva genera apoyo hacia los partidos políticos en el poder, incrementa la competitividad electoral de dichos partidos y de las personas en el gobierno y, de manera general, fortalece la democracia (Shultz, 1995; Arana y Caicedo, 2013).

La buena reputación gubernamental ayuda a los gobiernos a legitimarse revirtiendo el public cynism o apatía ciudadana hacía la política y aumentando los niveles de confianza en el gobierno y las autoridades, además de incrementar la participación ciudadana (Da Silva y Batista, 2007).

Se puede afirmar que tanto legitimidad como reputación son percepciones de aprobación de las acciones de un gobierno; la segunda genera una diferenciación positiva respecto a otras organizaciones (King y Whetten, 2008).

\subsection{Los factores determinantes de la reputación gubernamental}

Una vez analizados los potenciales beneficios de que un gobierno posea buena reputación, es necesario definir de qué depende, es decir, cuáles son los factores o variables que la determinan. De la revisión de la literatura se encontró que los principales factores fueron el desempeño gubernamental, el compromiso ético, el liderazgo de las autoridades y la comunicación gubernamental

\section{3. 1. Desempeño gubernamental}

Esta variable se caracteriza por el cumplimiento de objetivos en relación con las necesidades de los ciudadanos, en prestar servicios públicos de calidad y en mejorar la calidad de vida implementando políticas adecuadas (Morales, 2010). Dentro del desempaño gubernamental englobamos tres dimensiones: la eficacia del gobierno en relación con los servicios municipales, la eficiencia en relación con recursos gastados y servicios prestados y el avance económico generado en el lugar (Reputation Institute, 2017).

\section{3. 2. Compromiso ético}

Esta variable de la reputación gubernamental se compone de dos dimensiones: la rendición de cuentas y el combate a la corrupción. La reputación se basa en la transparencia, existencia del estado de derecho, partici- 
pación ciudadana, rendición de cuentas y responsabilidad (Agere, 2000); sin lugar a dudas, respetar la ley y que un gobierno sea responsable con el entorno también abonan a una buena reputación (Passow et al., 2005).

Eisenegger (2008), al describir las tres dimensiones de la reputación, habla sobre la dimensión social y la asocia con el mundo de lo normativo, de lo bueno, esto quiere decir que la organización debe cumplir con ciertos estándares, ser íntegra y tener responsabilidad social. Se puede afirmar que una organización que busca mejorar sus niveles de reputación debe cumplir con ciertos estándares y ser íntegra, y una manera de lograrlo es siendo transparente y cumpliendo la Ley. Por otro lado, existe una asociación positiva entre el nivel de transparencia gubernamental y la reputación del país, por lo que a mayor transparencia mayor reputación (Beuren y Almeida-Santos, 2013).

Es imperativo que existan instituciones íntegras, responsables, confiables y que cumplan con la ley. También, es necesario que el gobierno realice acciones para disminuir los actos de corrupción y que autoridades y particulares reciban las sanciones establecidas cuando se cometan dichos actos (Arana y Caicedo, 2013; Fombrun, 1996; Eisenegger, 2008).

\section{3. 3. Liderazgo de las autoridades}

Esta variable se basa en la capacidad que tienen para transmitir con claridad los objetivos de su gobierno, así como en el compromiso con la institución; ambos atributos son vitales para la gestión de reputación (LGA, 2013). De acuerdo con Fombrun y Van Riel (2003) la visión de la organización y el liderazgo de sus directivos son esenciales en la gestión de reputación.

Un gobierno con buena reputación debe tener autoridades que inspiren a otros con una dirección y propósitos claramente articulados; se considera vital el liderazgo del alcalde y otros miembros del ayuntamiento (LGNZ, 2015).

De igual modo, son fundamentales para la gestión de una buena reputación la autoridad, carisma y estima que poseen los liderazgos, así como que sepan comunicarse de manera efectiva con los diferentes stakeholders (Luoma-aho, 2008; Passow et al., 2005).

\section{3. 4. Comunicación gubernamental}

La información es vital en un modelo de democracia en el que se espera que los ciudadanos participen en la toma de decisiones siempre y cuando parezcan racionales (Dalton y Wattenberg, 1993). La política se basa en procesos de comunicación con individuos; por ello, la democracia no puede existir sin comunicación y los gobiernos deben ser conscientes de ello (Sánchez, 2004).

Esta variable implica distintas dimensiones tales como la comunicación externa y las relaciones públicas, la comunicación al interior del gobierno, la innovación en mecanismos de información, escuchar y actuar conforme a las demandas de los ciudadanos y, por último, branding o la generación de marca gobierno.

La comunicación gubernamental tiene el objetivo de informar a los ciudadanos de la posición que tienen los gobiernos y de las políticas públicas, así como de legitimar la acción política y crear consentimiento público (Pfetshe, 2008). Para tener una buena reputación es necesario comunicar las fortalezas y atributos positivos de la organización (Canel, 2014).

No se trata únicamente de comunicar y utilizar técnicas de marketing político para generar reputación. No se puede crear una imagen o marca que no esté basada en una realidad, ya que se crea una falsa concepción con consecuencias negativas potenciales (Loo y Davies, 2006).

Una vez que se conocieron los factores, que de acuerdo con la literatura revisada determinan la reputación gubernamental, en el siguiente apartado se analiza la metodología para ponderar todos los factores de la reputación. 


\section{Metodología}

La metodología empleada en el artículo utiliza el proceso analítico jerárquico (AHP, Analytic Hierarchy Process), también conocido como algoritmo de Saaty. Este proceso analítico jerárquico es una metodología de análisis multicriterio desarrollada por Thomas Saaty en la década de los setenta en el cual se obtienen valores numéricos para juicios de preferencia y los sintetiza para determinar la prioridad de cada una de las variables (Gallardo, 2012).

Saaty (2008) explica las etapas del proceso analítico jerárquico. En primer lugar, es necesario establecer criterios o alternativas que tiene el problema; posteriormente, la recolección de información puede ser obtenida de expertos o de tomadores de decisiones, con esta información se darán prioridades entre las variables estableciendo si hay una relación entre parejas de variables ya sea de igualdad, ligeramente más fuerte, muy fuerte o extremadamente fuerte respecto a la otra; por último, con la información recolectada se construye una matriz de comparación recíproca para establecer cuál es el valor numérico de cada una de las variables. En este apartado se detalla el proceso del AHP en cinco pasos:

\section{1. Selección de literatura}

Para realizar el AHP, Saaty (2008) recomienda que la relación entre las frecuencias se realice con base en expertos en el tema. Para este trabajo se decidió que más de treinta obras especializadas en reputación en el sector público sirvieran de guía para determinar las variables, sus dimensiones y posteriormente la relación entre ellas. El criterio de selección parte del análisis de autores de la revista científica principal en tema de reputación a partir del 2000: Corporate Reputation Review, la cual presenta algunos artículos sobre la importancia de los intangibles; aunque se enfoca en el sector privado, presenta algunos autores que comienzan con su análisis en el sector público; otro punto de partida fue el International Journal of Public Sector Management, otros referentes que se utilizaron fueron los casos prácticos de gobiernos en el mundo que hoy en día ya cuentan con mecanismos de medición y gestión de reputación gubernamental. Lamentablemente, no existen revistas científicas especializadas sobre reputación en el sector público. La literatura analizada para el proceso analítico jerárquico se encuentra en el anexo.

\section{2. Análisis de frecuencia de variables y dimensiones}

De la revisión y análisis de la literatura se encontraron cuatro variables independientes con sus respectivas dimensiones. Se elaboró una tabla de frecuencias utilizando un mecanismo binario en el que se otorgó 0 a la variable cuando no aparece en la literatura y 1 cuando sí aparece. Con esto se logró establecer un orden por relevancia de cada una de las dimensiones.

En la tabla 1 se presenta el orden que obtuvieron las dimensiones de la reputación gubernamental con relación a la frecuencia.

\section{3. Asignación de valores con base en la escala de Saaty}

Una vez elaborada la tabla de frecuencias de las variables y dimensiones se observó cuáles son las más mencionadas en la literatura como factores determinantes de la reputación y se procedió a asignar valores de acuerdo con la escala de Saaty. Este método consiste en establecer la importancia comparativa entre cada par de alternativas a través de una escala determinada de valores que van del 1 al 9 (Gallardo, 2012). La tabla 2 muestra esta escala de valores que explican la relación de las variables. 
TABLA 1

Orden de frecuencias para asignar valores en la escala de Saaty

\begin{tabular}{|lc|}
\hline \multicolumn{1}{|c}{ Dimensiones de variable } & Orden por frecuencia \\
\hline Eficacia gubernamental & 1 \\
Eficiencia gubernamental & 8 \\
Avance económico & 4 \\
Transparencia y rendición de cuentas & 6 \\
Combate a la corrupción & 8 \\
Liderazgo de las autoridades & 8 \\
Comunicación externa y relaciones públicas & 2 \\
Comunicación interna & 3 \\
Innovación en mecanismos de información & 11 \\
Escuchar y actuar & 5 \\
Branding & 7 \\
\hline
\end{tabular}

Fuente: elaboración propia.

Con base en la escala numérica para jerarquizar variables (tabla 2) se asignaron valores a cada par de dimensiones. El punto de partida es la alternativa que tuvo más menciones en contraste con todas las demás en la tabla de frecuencia de literatura; por ejemplo, eficacia gubernamental se mencionó más respecto a innovación en mecanismos de información, que tuvo la menor cantidad de menciones y recibirá un valor de 9 , es decir, la eficacia gubernamental es extremadamente preferida en relación con la innovación en mecanismos de información. Una vez asignados los valores para cada una de las parejas de dimensiones, se procedió a llenar la matriz de comparación de variables, véase cuadro 1.

TABLA 2

Escala de Saaty Jerarquización de variables

\begin{tabular}{|c|c|c|}
\hline \multicolumn{3}{|r|}{ Jerarquización de variables } \\
\hline $\begin{array}{l}\text { Escala } \\
\text { numérica }\end{array}$ & Escala verbal & Explicación \\
\hline 1 & Igualmente preferida & Dos elementos contribuyen en igual medida al objetivo \\
\hline 2 & & Valor intermedio \\
\hline 3 & Moderadamente preferida & La experiencia y juicio favorecen levemente a un elemento sobre otro \\
\hline 4 & & Valor intermedio \\
\hline 5 & Fuertemente preferida & La experiencia y juicio favorecen fuertemente a un elemento sobre otro \\
\hline 6 & & Valor intermedio \\
\hline 7 & $\begin{array}{l}\text { Preferencia muy fuerte o } \\
\text { demostrada }\end{array}$ & $\begin{array}{l}\text { Un elemento es mucho más favorecido que el otro, predominancia } \\
\text { demostrada en la práctica }\end{array}$ \\
\hline 8 & & Valor intermedio \\
\hline 9 & Extremadamente preferida & La evidencia que favorece una sobre la otra es la más alta posible \\
\hline $\begin{array}{l}\text { Valores } \\
\text { recíprocos }\end{array}$ & $\begin{array}{l}\text { Cuando se asigna uno de lo } \\
\text { valor recíproco. }\end{array}$ & valores anteriores al elemento $i$ respecto de $j$, el elemento $j$ tendrá el \\
\hline
\end{tabular}

Fuente: Saaty, 2008. 


\subsection{Generación de la matriz de Saaty}

Pacheco y Contreras (2008) explican que la finalidad de la matriz dentro del proceso analítico jerárquico es establecer qué tanto importa una alternativa respecto a otra.

Este procedimiento inicia con la dimensión que tuvo el mayor número de menciones; como ejemplo se explica la comparación de la alternativa 1 respecto a sí misma, por lo cual recibe el código 1 que significa que son igualmente preferidas. Continuamos con la comparación de la alternativa 1 respecto a la 8 ; se le asigna el código 8 que significa que es entre muy fuerte y extremadamente preferida, y así se sigue con toda la fila. A la par se pueden ir completando las casillas recíprocas; por ejemplo, la dimensión 8 en relación con la 1 cuyo valor será el recíproco, es decir $1 / 8$.

El cuadro 1 muestra la matriz de comparación de alternativas terminada y después se procede al proceso de normalización de la matriz que se presenta en el punto siguiente. El número de las dimensiones que aparece en la primera columna y primera fila es el orden que cada dimensión tuvo en cuanto a las menciones en la tabla de frecuencia de la literatura (tabla 1 ).

\section{CUADRO 1}

Matriz de comparación de alternativas

\begin{tabular}{|l|c|c|c|c|c|c|c|c|c|c|c|}
\hline \multicolumn{10}{|c|}{ Matriz escala Saaty } \\
\hline & 1 & 8 & 4 & 6 & 8 & 8 & 2 & 3 & 11 & 5 & 7 \\
\hline 1 & 1 & 8 & 4 & 6 & 8 & 8 & 2 & 3 & 9 & 5 & 7 \\
\hline 8 & $1 / 8$ & 1 & $1 / 5$ & $1 / 3$ & 1 & 1 & $1 / 7$ & $1 / 6$ & 2 & $1 / 4$ & $1 / 2$ \\
\hline 4 & $1 / 4$ & 5 & 1 & 3 & 5 & 5 & $1 / 3$ & $1 / 2$ & 6 & 2 & 4 \\
\hline 6 & $1 / 6$ & 3 & $1 / 3$ & 1 & 3 & 3 & $1 / 5$ & $1 / 4$ & 4 & $1 / 2$ & 2 \\
\hline 8 & $1 / 8$ & 1 & $1 / 5$ & $1 / 3$ & 1 & 1 & $1 / 7$ & $1 / 6$ & 2 & $1 / 4$ & $1 / 2$ \\
\hline 8 & $1 / 8$ & 1 & $1 / 5$ & $1 / 3$ & 1 & 1 & $1 / 7$ & $1 / 6$ & 2 & $1 / 4$ & $1 / 2$ \\
\hline 2 & $1 / 2$ & 7 & 3 & 5 & 7 & 7 & 1 & 2 & 8 & 4 & 6 \\
\hline 3 & $1 / 3$ & 6 & 2 & 4 & 6 & 6 & $1 / 2$ & 1 & 7 & 3 & 5 \\
\hline 11 & $1 / 9$ & $1 / 2$ & $1 / 6$ & $1 / 4$ & $1 / 2$ & $1 / 2$ & $1 / 8$ & $1 / 7$ & 1 & $1 / 5$ & $1 / 3$ \\
\hline 5 & $1 / 5$ & 4 & $1 / 2$ & 2 & 4 & 4 & $1 / 4$ & $1 / 3$ & 5 & 1 & 3 \\
\hline 7 & $1 / 7$ & 2 & $1 / 4$ & $1 / 2$ & 2 & 2 & $1 / 6$ & $1 / 5$ & 3 & $1 / 3$ & 1 \\
\hline
\end{tabular}

Fuente: elaboración propia con base en Mercado (1991).

\section{5. Normalización de la matriz de Saaty}

Una vez completada la matriz de comparación de alternativas es necesario normalizarla El cuadro 2 muestra el proceso de normalización para obtener el porcentaje de cada una de las variables independientes y sus dimensiones respecto a la variable dependiente. Mercado (1991) explica los pasos para obtener los porcentajes de cada una de las variables y dimensiones y que se describe a continuación:

a) Se multiplican todos los factores entre sí de cada una de las filas; por ejemplo, en la primera fila se multiplica (cuadro 2):

$$
(1)(8)(4)(6)(8)(8)(2)(3)(9)(5)(7)=23224320
$$


Este resultado se encuentra en la columna con un número 1 del cuadro 2. Se repite el mismo procedimiento en cada una de las filas de la matriz y se colocan en la columna con el número 1.

\section{CUADRO 2}

Matriz normalizada de variables

\begin{tabular}{|lrccc}
\hline Alternativa & \multicolumn{1}{c}{ Normalización } & $\mathbf{2}$ & Porcentaje & Porcentaje agrupado \\
\hline 1 & 23224320 & 4.673380934 & 27.73108 & \\
2 & $4.9603 \mathrm{E}-05$ & 0.406146467 & 2.410007 & 40.97299811 \\
3 & 750 & 1.825446906 & 10.83191 & \\
4 & 0.3 & 0.896325121 & 5.318648 & 7.728655248 \\
5 & $4.9603 \mathrm{E}-05$ & 0.406146467 & 2.410007 & 2.410007289 \\
6 & $4.9603 \mathrm{E}-05$ & 0.406146467 & 2.410007 & \\
7 & 987840 & 3.50728865 & 20.81168 & \\
8 & 30240 & 2.554615936 & 15.15868 & \\
9 & $6.8893 \mathrm{E}-07$ & 0.275317787 & 1.633691 & 48.88833936 \\
10 & 16 & 1.286664898 & 7.634861 & \\
11 & 0.0047619 & 0.615019881 & 3.649428 & \\
\hline Total & & $\mathbf{1 6 . 8 5 2 4 9 9 5 1}$ & $\mathbf{1 0 0}$ & \\
\hline
\end{tabular}

Fuente: elaboración propia con base en Mercado (1991).

b) Posteriormente, se obtiene la raíz $11 \sqrt{ }$, puesto que hay 11 alternativas, a cada uno de los resultados de la columna con el número 1 :

$$
11 \sqrt{(23224320)}=4.673380934
$$

El resultado de dicho procedimiento se encuentra en la columna con el número 2 y se repite para cada uno de las filas.

c) Se procede a hacer la sumatoria de los resultados de las raíces $11 \sqrt{ }$ que se encuentran en la columna con el número 2: el resultado es 16.85249951.

d) Para obtener el porcentaje de cada una de las alternativas, se multiplica el resultado de la raíz $11 \sqrt{ }$, que se encuentra en la columna con el número 2, por 100, esto dividido entre la sumatoria de las raíces, por ejemplo en la primera fila:

$$
\frac{(4.6733800934)(100)}{16.85249951}=27.7310848
$$

El resultado anterior implica que la alternativa 1 determina a la reputación gubernamental en un 27.73\%. Esta operación se repite para cada una de las filas y así se obtiene el porcentaje de cada una de las alternativas.

e) Por último, se agrupan las alternativas por variable para ver el porcentaje de cada una, el cual se expresa en la columna de porcentaje agrupado. En el caso de las alternativas o dimensiones que conforman la variable $\mathrm{X} 1$, el desempeño gubernamental, se suman los siguientes valores: 


$$
(27.7310848)+(2.41000729)+(10.831906)=40.97299811
$$

Podemos concluir que la variable X1, desempeño gubernamental, tiene un valor de $40.97 \%$ respecto a la variable dependiente reputación gubernamental.

\section{Resultados}

Una vez realizado el proceso analítico jerárquico para el caso concreto de las variables y dimensiones de la reputación gubernamental, es pertinente analizar en qué medida impacta a la reputación gubernamental cada una de éstas. La figura 1 presenta de manera gráfica la jerarquía y distribución de cada variable independiente, así como de sus dimensiones respecto a la variable dependiente, que es la reputación gubernamental.

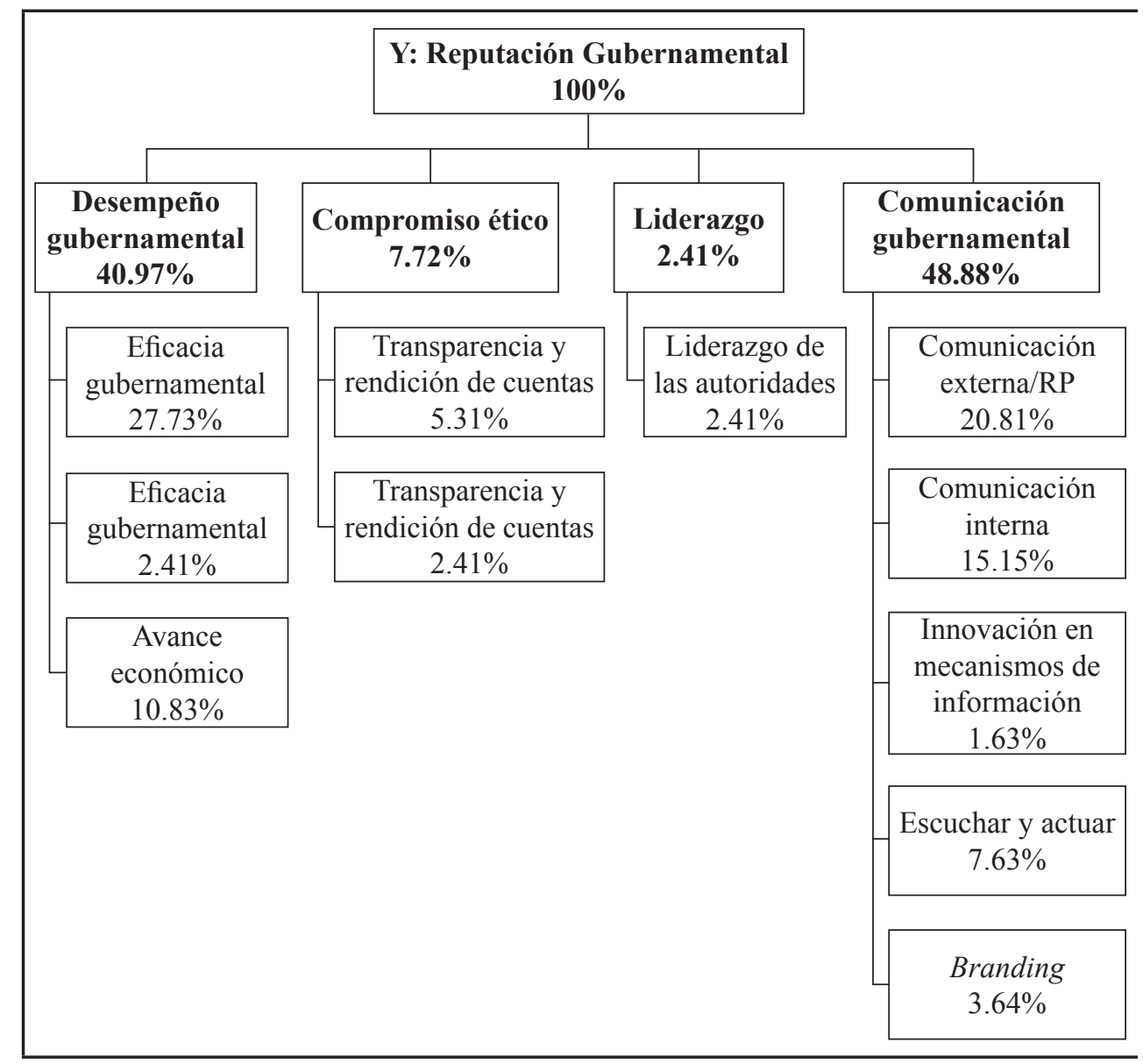

FIGURA 1

Diagrama jerárquico de variables y dimensiones

Fuente: elaboración propia con base en Gallardo (2012) y Mercado (1991).

Partimos de la variable dependiente con el mayor valor, que es la comunicación gubernamental, la cual impacta en $48.88 \%$ a la reputación gubernamental. Como se mencionó en la revisión de la literatura, esta variable se compone de cinco dimensiones, donde destaca la dimensión de comunicación externa y relaciones públicas, que tiene un impacto de $20.81 \%$, sigue la dimensión comunicación interna con un $15.15 \%$, la de escuchar y actuar con un $7.63 \%$, la de branding con un 3.64\% y la dimensión de innovación en mecanismos de información en un $1.63 \%$. 
De acuerdo con estos resultados, la forma como un gobierno se comunique con sus stakeholders externos y cómo las estrategias de relaciones públicas sean con ellos define la dimensión más valiosa; cabe resaltar que la comunicación con stakeholders internos no ocupa un valor poco importante, es decir, si bien hay que cuidar a los stakeholders externos, también aquellos al interior de un gobierno son importantes, ya sean autoridades electas, empleados de confianza, servidores públicos de carrera o sindicalizados.

Es primordial que un gobierno comprenda el proceso de comunicación no sólo como difusión de logros e información sino también como un proceso de escucha activa de los stakeholders. El valor de la dimensión escuchar y actuar es significativo y sin duda debe tomarse en cuenta.

Se puede observar a partir del análisis realizado que hay dos dimensiones con valores menores: la primera enfocada en la marca gobierno y la segunda en contar con mecanismos de información innovadores; es probable que estos últimos sean concebidos dentro de las dos dimensiones de comunicación, pero será necesaria una mayor investigación para definirlo.

En segundo lugar se encuentra la variable desempeño gubernamental, con un valor de $40.97 \%$ y que comprende tres dimensiones, entre ellas sobresale la dimensión eficacia gubernamental con un valor de $27.73 \%$, es decir, la dimensión con mayor valor y por ende con mayor impacto en la gestión de reputación gubernamental. Dentro de esta segunda variable también encontramos la dimensión avance económico con un valor de $10.83 \%$ y la de eficiencia gubernamental con $2.41 \%$.

Vale la pena poner de relieve la importancia de la eficacia gubernamental, es decir, de la prestación de servicios públicos, ejecutar bien las tareas que son su razón de ser; en el caso de un municipio, prestar al menos los servicios públicos que son su obligación por mandato legal. Sin duda, partiendo de la literatura, las condiciones económicas favorables en un lugar determinado son un factor a considerar en la gestión de reputación.

La dimensión que pareciera poco importante es la eficiencia gubernamental, es decir, el gasto eficiente de recursos en relación con los servicios prestados; es conveniente que se profundice en el análisis de esta dimensión, ya que el valor obtenido se puede deber a que los autores se desarrollan en otras realidades políticas y económicas en donde ser eficiente en los recursos públicos se da por sentado.

En tercer lugar está la variable independiente compromiso ético con un valor de $7.72 \%$, dentro de la cual la dimensión transparencia y rendición de cuentas tiene un valor de $5.31 \%$; con base en el ejercicio realizado, que el gobierno sea transparente y rinda cuentas sólo impacta un $5.31 \%$ en su reputación gubernamental. Por su parte, la dimensión combate a la corrupción tiene un valor de $2.41 \%$, lo cual hace pensar a priori que no sería un factor determinante.

Sin duda alguna, es trascendental el análisis profundo de la variable compromiso ético en futuras investigaciones y entender que la mayoría de la bibliografía desarrollada en gestión de intangibles en el sector público se realiza en países europeos y en Estados Unidos, lo cual puede sugerir que se encuentren en otra realidad política donde el combate a la corrupción y a la transparencia son problemáticas muy distintas a la realidad mexicana.

Por último, analizamos la variable independiente liderazgo de las autoridades que tiene un valor del $2.41 \%$ y deja ver que es un factor poco importante en la gestión de la reputación; sin embargo, de la revisión de literatura se puede desprender que el liderazgo de las autoridades es un atributo necesario para lograr un gobierno eficiente y que comunique adecuadamente.

Una vez que se conoce el impacto de cada una de las variables independientes y sus dimensiones en relación con la reputación gubernamental, es valioso entender que esto proporciona directrices para aquellos gobiernos que desean gestionar una reputación positiva. Como punto de partida, un gobierno tendrá que trabajar arduamente en prestar servicios públicos de calidad y en comunicarse de manera eficiente con sus stakeholders para entender sus necesidades y expectativas para actuar en consecuencia. 


\section{Conclusiones y ANÁLISIS PROSPECTIVO}

De acuerdo con lo presentado en este artículo, se puede argumentar que la reputación es un intangible multifactorial que por un lado depende de la percepción y por otro de las experiencias de múltiples stakeholders con la organización; sin embargo, lo importante es cómo una organización va a gestionar esa reputación.

En este sentido, entre los principales beneficios políticos, sociales y económicos de la reputación destaca que la reputación gubernamental positiva genera inversión, aumenta la atracción turística, incrementa el capital social y la participación ciudadana con el gobierno.

La reputación gubernamental al ser un intangible no sólo multifactorial sino también multi-stakeholer vuelve complejo el proceso de identificación de factores determinantes o variables independientes; sin embargo, del análisis de la literatura se encontró que el desempeño gubernamental, el compromiso ético, el liderazgo de las autoridades y la comunicación gubernamental son los factores que determinan la reputación gubernamental. Cada una de estas variables independientes cuenta con dimensiones que nos permiten entender mejor cómo es que un gobierno puede gestionar su reputación.

El proceso analítico jerárquico permitió la ponderación de las variables y sus dimensiones conociendo en qué medida determinan la reputación; es notable que la base para ponderar y priorizar las variables y dimensiones es el estudio de literatura relevante sobre reputación en el sector público.

Se puede concluir que las variables que inciden en mayor medida en la reputación gubernamental son el desempeño y la comunicación gubernamentales. Después de la ponderación analítica se observa que éstas determinan la reputación gubernamental en un $89.85 \%$, mientras que el resto de las variables sólo la explican en un $10.15 \%$.

Como recomendación para autoridades y servidores públicos en los gobiernos, cabe señalar que la reputación si bien es un intangible que recae en el juicio de terceros, depende de las acciones del gobierno y se puede gestionar.

Una de las principales recomendaciones para los gobiernos es prestar servicios públicos de calidad, por lo que será tarea de los gobiernos acordar qué servicios públicos son los más importantes para el ciudadano; lo anterior no porque se puedan eximir de su obligación legal, política y moral de prestarlos sino para poner especial atención en aquellos que accionan una serie de percepciones y experiencias positivas en los stakeholders.

Los gobiernos deben trabajar y generar condiciones de avance y crecimiento económico para que exista seguridad e inversión, que el marco normativo sea sencillo, transparente y que facilite la apertura de negocios y se generen empleos.

Es vital que los gobiernos den el valor debido a la comunicación gubernamental y la dejen de ver como un área de difusión y enlace con medios de comunicación; es imprescindible que los gobiernos tengan profesionales que sean capaces de transmitir a sus stakeholders internos los valores, misión y visión de la organización y que también tengan capacidad de diseñar estrategias para escuchar y tomar en cuenta en la toma de decisiones a los diferentes grupos de stakeholders, tanto a los que se encuentran en la ciudad o jurisdicción gubernamental como con los stakeholders de otros lugares.

La comunicación gubernamental no puede ser una estrategia aislada o disonante de la realidad que experimentan los ciudadanos y otros stakeholders, puesto que los gobiernos deben tener claro que no se debe ni se puede comunicar lo que no se hace, ya que sólo generará más apatía, niveles menores de aprobación, hartazgo y desencanto político.

Cómo futuras líneas de investigación se plantea la necesidad de calibrar el valor de las variables en el contexto nacional para que los resultados del proceso de ponderación analítica respondan a las características y condiciones de México, y no nada más con base en la literatura que si bien brinda directrices de los factores determinantes, atienden a una realidad social, cultural y política distinta. Valdría la pena utilizar otra fuente para la ponderación de variables y que no sólo parta de un análisis de literatura sino de una investigación cualitativa con diferentes stakeholders. 


\section{Agradecimientos}

Se agradecen los comentarios de los árbitros de la revista que mejoraron sustancialmente el contenido del artículo.

\section{REFERENCIAS}

Agere, S. (2000). Promoting good governance. Principles, practices and perspectives. London: Commonwealth Secretariat.

Arana, R. y Caicedo, G. (2013). La reputación gubernamental. Una aproximación pragmática a su análisis. Saarbrücken: Editorial Académica.

Bannister, F., Remenyi, D. y Batista, L. (2003). Potentialities of customer relationship management in the building of government reputation. In F. Bannister \& D. Remenyi (Eds.), Proceedings of the Third European Conference on e-Government (pp. 1-11). Dublin: ECEG.

Beuren, I., \& Almeida-Santos, P. (2013). Relationship between government transparency and reputation. Globalization, Competitiviness and Governabilitu Journal, 7(3), 89-102.

Bussy, N., \& Kelly, L. (2010). Stakeholders, politics and power towards an understanding of stakeholder identification and salience in government. Journal of Communication Management, 14(49), 289-305.

Cahill, M., Batista, L. y Kawalek, P. (2004). The recovery of government reputation: Exploring two dimensions of strategy. AMCIS 2004 Proceedings. Retrieved from http://aisel.aisnet.org/amcis2004/133

Canel, M. y García, A. (2013). Communicating trusting governments, Analysis of trust as an intangible asset of the Spanish central government. Zer, 18(34), pp. 29-48.

Canel, M. (2014). Reflexiones sobre la reputación ideal de la administración, en M. Herrero et al. (Eds.), Escribir en las almas. Estudios en honor de Rafael Alvira (pp. 69-88). Pamplona: Eunsa.

Canel, M. (2017). La investigación sobre los bienes intangibles en la administración pública, en M. Canel, P. Piqueiras y G. Ortega (Eds.), La comunicación de la administración pública: conceptos y casos prácticos de bienes intangibles (pp. 11-28). Madrid: Instituto Nacional de Administración Pública.

Colmar-Brunton. (2014). Local Government Reputation Index, Waitomo Distric Council Public Boost. Nueva Zelanda: Colmar Brunton.

Consulta Mitofsky. (2016). Enrique Peña Nieto a 15 Trimestres de Gobierno. Consulta Mitofsky. Consultado el 16 de noviembre de 2016. Disponible en file://Users/joanna/Downloads/201608_NA_EVGOB_TRIM15.pdf

Dalton, R., \& Wattenberg, M. (1993). The not so simple act of voting. In A. Finifter (Ed.) Political Science. The State of the Discipline II (pp. 193-218). Washington: American Political Science Association.

Da Silva, R., \& Batista, L. (2007). Boosting government reputation through CRM. International Journal of Public Sector Management, 20(7), 588-607.

Eisenegger, M., \& Imhof, K. (2008). The True, the good and the beautiful: Reputation management in the media society. In A. Zerfass B. Ruler K. Sriramesh (Eds.), Public Relations Research. European and International Perspectives and Innovations (pp. 125-146).VS Verlag für Sozialwissenschaften: Wiesbaden.

Eisenegger, M. (2008). Reputation in Politics. In Kaid, L., \& Holtz-Bacha, C. (Eds.), Encyclopedia of Political Communication (pp. 712-714). London: SAGE. 
Fombrun, C. (1996). Reputation, realizing value from the corporate image. Massachusetts: Harvard Business School Press.

Fombrun, C., \& Shanley, M. (1990). What's a name? Reputation building and corporate strategy. Academy of Management Journal, 33(2), 233-258.

Fombrun, C., \& Van Riel, C. (2003). Fame\&fortune: How successful companies build winning reputations. New Jersey: Prentice Hall.

Fullerton, J. y Holtzhausen, D. (2012). Americans' attitudes toward South Africa: A study of country reputation and the 2010 FIFA World Cup. Place Branding and Public Diplomacy, 8(4), 269-283.

Gallardo, J. (2012). Administración estratégica, de la visión a la ejecución. México: Alfaomega.

GCN. (2014). Working with stakeholders. United Kingdom: Local Government Association.

King, B., \& Whetten, D. (2008). Rethinking the relationship between reputation and legitimacy: A social actor conceptualization. Corporate reputation Review, 11(3), 192-207.

Kizil, C., \& Atalan, A. (2015). Image and Reputation of Yalova City: A study on Yalova University Students. Emerging Market Journal, 5(2).

LGA. (2008). The reputation of local government, literature review to support “my council campaign”. United Kingdom: Local Government Association.

LGA. (2013). New reputation guide. United Kingdom: Local Government Association. Retrieved from http:// reputation.lga.gov.uk/lga/core/page.do?pageId $=11430566$

LGNZ. (2015). Building a stronger local government for New Zealand. A survey of New Zealandersperception of local government. New Zeland: Local Government Association. Retrieved from http://www.lgnz.co.nz/ assets/In-background/LGNZ-2015-Survey-Report-FINAL2.pdf

Loo, T., \& Davies, G. (2006). Branding China: The ultimate challenge in reputation management? Corporate Reputation Review, 9(3), 198-210.

Luoma-aho, V. (2007). Neutral reputation and public sector organization. Corporate Reputation Revien, 10(2), 124-143.

Luoma-aho, V. (2008). Sector reputation and public organizations. International Journal of Public Sector Management, 21(5), 446-467.

Luoma-aho, V. (2011). Do expectations shape public sector reputation? In A. Arild Wæraas, H. Byrkjeflot, \& S. I. Angell (Eds.), Substans og Framtreden. Omdommehaindtering i offentlig sektor. Universitetsforslaget: Oslo. Retrieved from https://www.academia.edu/954150/Do_expectations_shape_public_sector_reputation?auto $=$ download.

Luoma-aho, V., \& Makikangas, E. (2014). Do public sector mergers (re)shape reputation? International Journal of Public Sector Management, 27 (1), 39-52.

Luoma-aho, V., \& Canel, M. (2016). Public sector reputation. In C. Carroll (Ed.), The Sage Encyclopedia of Corporate Reputation (pp. 597-600). Sage Publications. Thousand Oaks, CA.

Mercado, E. (1991). Técnicas para la toma de decisiones. México: Noriega Editores.

Morales, G. (2010). Evaluación del desempeño gubernamental y cultura organizacional: Reflexiones desde la Nueva Gestión Pública y la sociología de las organizaciones. Revista de Administración Pública, 45(1), pp. 37-52.

Pacheco, J. y Contreras, E. (2008). Manual metodológico para evaluación multicriterio para programas y proyectos. Santiago de Chile: ILPES-CEPAL.

Passow, T., Fehlmann, R., \& Grahlow, H. (2005). Country Reputation-From Measurement to Management: The Case of Liechtenstein. Corporate Reputation Review, 7(4), 309-328. 
Pfetsche, B. (2008). Government Communication. In L. Kaid \& C. Holtz-Bacha (Eds.), Encyclopedia of Political Communication (pp. 279-280). London: SAGE.

Quintero, C., \& Pérez, D. (2014). The Mexican Reputation, a strategy to improve the stereotype Mexico shares with its U.S. Diaspora. USA: Harvard University. Retrieved from http://ash.harvard.edu/files/ash/files/ mexican-reputation.pdf

Reputation Institute. (2016a). Most reputable countries in 2016. New York: Reputation Institute.

Reputation Institute. (2017). Most reputable cities in 2016. New York: Reputation Institute.

Romenti, S. (2010). Reputation and stakeholder's engagement: An Italian case study.Journal of Communication Management, 14(4), 306-318.

Sánchez, E. (2004). Comunicación y democracia. México: Instituto Federal Electoral.

Saaty, T. (2008). Decision making with the analytic hierarchy process. International Journal of Services Science, $1(1), 83-98$.

Shultz, K. (1995). The politics of the political business cycle. British Journal of Political Science, 25(1), 79-99.

Transparencia Mexicana y Consulta Mitofsky. (2004). Indice mexicano de reputación empresarial. Disponible en http://gda.itesm.mx/images/archivos/Mitofskiresumen.pdf

WVS. (2012). World Values Survey México 2012. Disponible en http://www.worldvaluessurvey.org/WVSDocumentationWV6.jsp

WVS. (2015). World Value Survey 2010-2014. Disponible en http://www.worldvaluessurvey.org/WVSDocumentationWV6.jsp

Yang, S., Lee, J., \& Wrigley, B. (2008). Country reputation in multidimensions: Predictors, effects and communication channels. Journal of Public Relations Research, 20(4), 421-440.

\section{BY-NC-ND}

